3 Iyer KS, Thampuran RVA. Beta blockade and anticonvulsant activity of propranolol. Ind Physiol Pharmacol 1978;Jul-Sep:293-6.

4 Kolbinger HM, Zierz S, Elger CE, Penin H. Startle-induced seizures and their relationship to epilepsy: three case reports. fournal ship to epilepsy: three
of Epilepsy 1990;3:23-7.

Methyl bromide induced neuropathy: a clinical, neurophysiological, and morphological study

Methyl bromide is a colourless, odourless gas used as a fumigant to disinfect soil, grains, and warehouses. Due to its high volatility, dangerous concentrations can accumulate rapidly in working areas. Inhalation is a main route of exposure. Dependent on dose and duration of exposure, methyl bromide can cause both acute CNS symptoms ${ }^{1}$ and chronic peripheral sensorimotor neuropathy..$^{2-4}$ Optic atrophy has also been described. ${ }^{5}$

A 23 year old Moroccan man working in Italy as a methyl bromide fumigator in greenhouses was admitted because of painful leg numbness. His personal history was unremarkable and he denied alcohol misuse. He had worked for the previous seven months (eight hours a day), wearing a protection mask irregularly. Neurological examination showed asymmetric lower limb weakness and areflexia, stepping gait, and sensory loss for pin prick, position, and vibration. Upper motor and cranial nerve signs were absent. Mental state was intact. Laboratory tests excluded diabetes, hepatic and collagen diseases, syphilis, tuberculosis, HIV, and Lyme related disease. Vitamin B12 and folic acid concentrations were normal. CSF was normal. Organophosphate and lead poisoning were excluded. One month after cessation of exposure urinary methyl bromide was $0.01 \mathrm{ppm}$. A colour vision test (Farnsworth-Munsell 100-hue test) showed defective perception of all colours, especially in the red-green axes. Eye fundi and evoked responses were normal.

Electrophysiology showed a severe motor neuropathy of axonal type confined to the legs. The right peroneal muscle action potential was unrecordable from the extensor digitorum brevis muscle. The tibial and left peroneal nerves had reduced motor conduction $(-2 \cdot 7$ and $-3 \cdot 1$ SD from mean control value) and muscle action potential amplitude $(0.2$ and $1.0 \mathrm{mV})$. Sensory findings in the sural nerve were normal. An EMG showed denervation and loss of motor units in distal muscles.

Tibialis anterior muscle biopsy showed neurogenic changes. A sural nerve sample was examined. Light microscopy showed that the endoneurial area and total fibre density were within the normal range. Multifocal, scattered loss of myelinated fibres (4689 $v$ 5200-9500 in normal subjects) was detected, with a prominent loss of fibres larger than $7 \mu \mathrm{m}$ (1321 $v$ 16503300 ), and relative sparing of the smaller axons. ${ }^{6}$ Degenerated fibres were seen. The frequency of clusters fell within the normal range and there was no inflammatory infiltrate. On teasing, $38 \%$ of the fibres had signs of segmental remyelination; $15 \%$ had shortening of all internodes, suggesting regeneration, whereas the incidence of demyelination multifocally distributed along the fibre length was $8 \%$. Electron microscopy showed ongoing degeneration of both

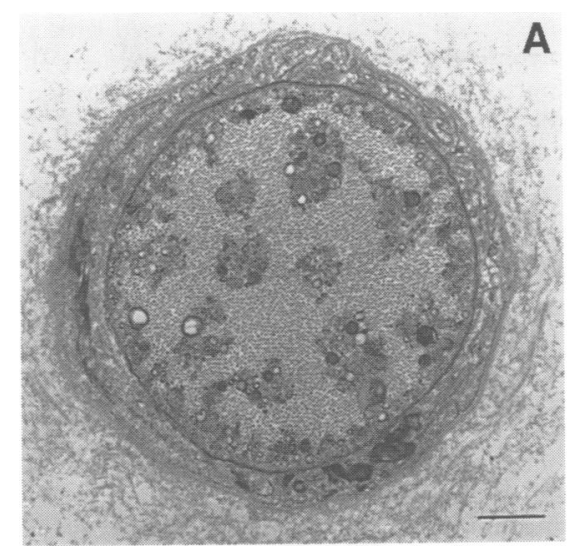

The presence of segregated microtubules in the nodes of Ranvier suggests that methyl bromide impairs axonal flow. As in most types of exogenous intoxication, methyl bromide may cause degeneration of axons with a dying back-like mechanism, more evident at the distal nodes. This neurotoxic agent may either alter the metabolism of the neuronal perikaryon or interfere with the dynamics of axonal transport. A metabolic mechanism for methyl bromide intoxication has recently been proposed. ${ }^{1}$ Based on neuropathological similarities with Wernicke's disease, methyl bromide intoxication has been correlated with an altered glycolysis and pyruvate oxidation.

Our patient and two healthy coworkers showed an acquired type dyschromatopsia. This may be a first sign of optic neuropathy, ${ }^{5}$ which precedes electrophysiological abnormalities. We therefore propose to use the Farnsworth-Munsell 100-hue test to screen workers exposed to methyl bromide.

We thank Mrs Laura Gandolfi for technical assistance.

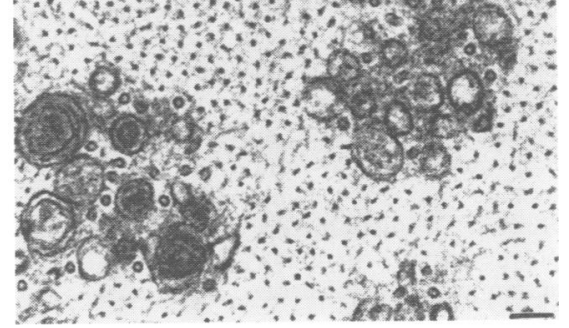

Figure (A) Electron micrograph showing a node of Ranvier with segregated microtubules. Calibration bar $=0.05 \mu \mathrm{m}$. (B) Segregated microtubules, altered mitochondria, dense bodies, and amorphous filamentous material are packed among masses of neurofilaments. Calibration bar $=0.1 \mu \mathrm{m}$.

myelinated and unmyelinated fibres. Numerous Schwann cell subunits were devoid of axons. Many nodes of Ranvier showed segregated axonal microtubules (figure).

The patient received $\mathrm{B} 1, \mathrm{~B} 6$, and $\mathrm{B} 12$ vitamins, folic acid, and amytriptiline (50 $\mathrm{mg} /$ day). When re-examined two months later, muscle strength was normal, whereas limb numbness was still present. Peroneal muscle action potential $(0.3 \mathrm{mV})$ was recordable and there were no signs of denervation. Two coworkers, similarly exposed to methyl bromide, had neither clinical nor electrophysiological signs of neuropathy, despite slightly altered colour vision.

The clinical history and laboratory data of this patient excluded known causes of peripheral neuropathy, suggesting that exposure to methyl bromide was responsible. The neurological time course corresponded with previous descriptions, where symptoms appeared after several months of exposure and the patients mostly recovered six to eight months after cessation of exposure. ${ }^{34}$

It has been proposed that methyl bromide causes a distal axonopathy. ${ }^{2}$ We report here morphological evidence for nerve abnormalities after exposure to methyl bromide. Loss of large axons and degeneration of both myelinated and unmyelinated fibres were found in the sural nerve. Spared myelinated and regenerating large fibres might account for the normal amplitude and maximal velocity of the sural action potential.

\section{Anti-D immunoglobulin treatment in chronic inflammatory demyelinating polyneuropathy}

Patients with immune thrombocytopenic purpura may have increases in platelet count after treatment with IVIg. The mechanism of action of intravenous 
immunoglobulin (IVIg) has been attributed to blockade of the receptors on the cell surface of macrophages and monocytes for the Fc region of IgG $(F c \gamma R)$ resulting in antibody dependent cellular cytotoxicity (ADCC) inhibition. ${ }^{1}$ This is supported by the findings that IVIg slows the clearance of red cells coated with anti-D immunoglobulin and treatment with anti-D raises the platelet count in D-positive patients with immune thrombocytopenic purpura with or without a spleen. Patients with GuillainBarré syndrome or chronic inflammatory demyelinating polyneuropathy may improve after plasma exchange, infusion of fresh frozen plasma, or after treatment with IVIg. ${ }^{2}$ Monocytes and macrophages play an important part in the pathogenesis of Guillain-Barré syndrome and chronic inflammatory demyelinating polyneuropathy. ${ }^{3}$ Inflammatory cells are present in the nerves from the earliest stages of GuillainBarré syndrome. Demyelination occurs only in tissue infiltrated with inflammatory cells and degenerated myelin is largely restricted to nerve fibres invaded by macrophages. ${ }^{4}$

To investigate if blockade of $F c \gamma R$ on invading macrophages by anti-D immunoglobulin results in improvement in chronic inflammatory demyelinating polyneuropathy, anti-D immunoglobulin was given to a D-positive patient with chronic inflammatory demyelinating polyneuropathy who had never had spontaneous remissions and who in a double blind crossover study had responded to IVIg and not to albumin. Response to anti-D immunoglobulin was compared with that after IVIg.

After approval by the ethics committee of the Academic Medical Centre and with the informed consent of the patient he received $26 \mu \mathrm{g} / \mathrm{kg}$ bodyweight/day intravenously on three consecutive days (total dose $3 \mathrm{mg}$ ) of human anti-D immunoglobulin (Rhesuman Berna iv). Haemoglobin fell from 10.5 $\mathrm{mmol} / 1$ on day 1 to $7.5 \mathrm{mmol} / \mathrm{l}$ on day 8 and the packed cell volume from 0.50 to $0 \cdot 34$. Haptoglobin decreased from $1.3 \mathrm{~g} / 1$ to values below $0 \cdot 1 \mathrm{~g} / 1$ on day 3 . From day 8 onwards these values slowly increased. Bilirubin increased from $6 \mu \mathrm{mol} / 1$ to 27 $\mu \mathrm{mol} / 1$ on day 3 . The erythrocyte direct antiglobulin test became strongly positive with a titre of $1: 256$ on day 3 .

After two weeks muscle strength, electrophysiological variables, and walking distance had not changed and treatment with $0.4 \mathrm{~g} / \mathrm{kg}$ bodyweight IVIg on five consecutive days was started. Muscle strength and walking distance improved. Most changes in electrophysiological measurements were in the direction of improvement.

The non-blind design has probably not influenced the results. The patient was very much in favour of this experimental treatment because of its convenience. The results of measurements of muscle strength during the first week after anti-D immunoglobulin treatment did not differ much. Moreover, electrophysiological testing, which cannot be influenced by the patient, showed changes in the direction of deterioration rather than improvement.

The anti-D immunoglobulin dosage was not too low. The same or lower dosage is effective in patients with immune thrombocytopenic purpura and haemolysis was clearly present. Improvement after subsequent IVIg treatment was convincingly shown by the increase in walking distance from 50 to at least $1000 \mathrm{~m}$ and by the increase in muscle strength as tested with a myometer. The results of measurement at four weeks showed that most were outside the mean +2 SD of the first results and most changes in electrophysiological measurements were in the direction of improvement although this method of testing is not very sensitive shortly after improvement in patients with chronic inflammatory demyelinating polyneuropathy.

Patients with immune thrombocytopenic purpura respond to anti-D and IVIg. The response to anti-D immunoglobulin is not restricted to patients with a spleen and enhanced ADCC blocking was found in anti-D preparations in an Ig-fraction distinct from the anti-D activity. The suggestion was that this strong ADCC inhibitory activity in anti-D immunoglobulin was due to Fc-receptor blocking antibodies produced in the course of immunisation.

This patient with chronic inflammatory demyelinating polyneuropathy responded to IVIg and not to anti-D. From this finding we conclude that either improvement after IVIg in chronic inflammatory demyelinating polyneuropathy and immune thrombocytopenic purpura is based on a common mechanism but that anti-D influences a pathogenic pathway in immune thrombocytopenic purpura which is not involved in chronic inflammatory demyelinating polyneuropathy or that IVIg has a different action in the two diseases.

\section{Nitric oxide production in bacterial} meningitis

Bacterial meningitis of adults is still a serious disease with the mortality from pneumococcal meningitis being as high as $30 \%$. Intracranial complications such as cerebrovascular involvement, brain oedema, and increased intracranial pressure may lead to irreversible neuronal damage. ${ }^{1}$ The pathophysiological mechanisms of bacterial meningitis are still unknown in detail Experimental studies of bacterial meningitis indicate that several mediators including cytokines, platelet activating factor, arachidonic acid metabolites, and reactive oxygen species are involved in the pathophysiology. ${ }^{2}$ Recently, we have shown that nitric oxide (NO) is involved as a mediator of cerebrovascular changes and brain oedema in the early phase of pneumococcal
We thank Professor Ph Rümke and Dr P Aalbersberg for the anti-D immunoglobulin (Primmed), Professor B Ongerboer de Visser for electrophysiological studies, and Dr E S Louwerse for measuring muscle strength.

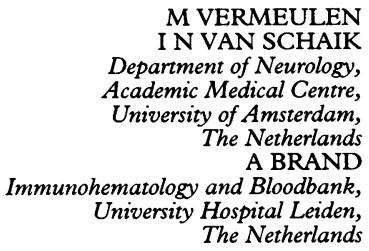

Correspondence to: Professor $M$ Vermeulen, Department of Neurology, AMC, Meibergdreef 9, 1105 AZ Amsterdam, The Netherlands.

1 Debré M, Bonnet MC, Fridman WH, et al. Infusion of $\mathrm{Fc} \gamma$ fragments for treatment of children with acute immune thrombocytopenic purpura. Lancet 1993;342:945-9.

2 Vermeulen $M$, Van der Meché FGA, Speelman JD, Weber A, Busch HFM. Plasma and gammaglobulin infusion in chronic inflammatory polyneuropathy. $\mathcal{F}$ Neurol Sci 1985;70:317-26.

3 Bansil S, Mithen FA, Singhal BS, Cook SD, Rohowsky-Kochan C. Elevated neopterin levels in Guillain-Barré syndrome. Arch levels in Guillain-Barr

4 Prineas JW. Pathology of the Guillain-Barre syndrome. Ann Neurol 1981;9(suppl):6-19.

5 Petri IB, Berek ALI. Further investigation of non-specific biological substance antiRh(D) preparations. Vox Sang 1986;51: 287-91.

meningitis in the rat. ${ }^{3}$ Nitric oxide, which is produced from L-arginine by NO-synthases (NOS) plays a part in the pathophysiology of a variety of CNS disorders including cerebral ischaemia and seizures and in inflammatory processes. ${ }^{4}$ During inflammation, large amounts of $\mathrm{NO}$ and superoxide radical are thought to be produced by polymorphonuclear leucocytes, macrophages, and blood vessels. We investigated NO production in serial CSF samples from six patients with bacterial meningitis (table). Production of NO was assessed by measuring nitrite, a stable metabolic product of NO. Nitrite determinations were made on $50 \mu \mathrm{l}$ aliquots of CSF sample mixed with $200 \mu \mathrm{l}$ of Griess reagent. The absorbance was read at $543 \mathrm{~nm}$ after 10 minutes of reaction and nitrite concentration was determined with reference to a standard curve from concentrations of $1 \mu \mathrm{M}$ to

White blood cell count (WBC) and nitrite concentration in CSF of six patients with bacterial meningitis

\begin{tabular}{|c|c|c|c|c|}
\hline $\begin{array}{l}\text { Patient } \\
\text { age/sex }\end{array}$ & $\begin{array}{l}\text { Days after } \\
\text { onset of } \\
\text { disease }\end{array}$ & $\begin{array}{l}\text { CSF WBC } \\
(\text { cells/ } / \mu l)\end{array}$ & $\begin{array}{l}\text { Bacteria cultured } \\
\text { from } C S F\end{array}$ & $\begin{array}{l}\text { CSF nitrite } \\
\text { (nmolll) }\end{array}$ \\
\hline \multirow[t]{3}{*}{$18 / \mathrm{F}$} & 1 & 3370 & Neisseria meningitidis & 57 \\
\hline & 2 & 1953 & - & 37 \\
\hline & 4 & 163 & - & 36 \\
\hline \multirow[t]{3}{*}{$34 / M$} & 1 & 2100 & Streptococcus pneumoniae & 85 \\
\hline & 2 & 1120 & - & 33 \\
\hline & 3 & 2730 & - & 36 \\
\hline \multirow[t]{3}{*}{$18 / \mathrm{M}$} & 1 & 23000 & Neisseria meningitidis & 69 \\
\hline & 16 & 140 & - & 25 \\
\hline & 66 & 33 & - & 27 \\
\hline \multirow[t]{2}{*}{$59 / \mathrm{F}$} & 1 & 1900 & Haemophilus influenzae & 34 \\
\hline & 5 & 61 & - & 24 \\
\hline \multirow[t]{3}{*}{$67 / F$} & 1 & 491 & Streptococcus pneumoniae & 47 \\
\hline & 2 & $22^{\star}$ & - & 44 \\
\hline & 6 & $10^{\star}$ & - & 42 \\
\hline \multirow{3}{*}{$60 / M$} & 1 & 9000 & Streptococcus bovis & 44 \\
\hline & 2 & 9813 & - & 24 \\
\hline & 4 & 336 & - & 27 \\
\hline
\end{tabular}

CSF nitrite concentrations in controls ranged from $20 \mathrm{nmol} / 1$ to $33 \mathrm{nmol} / 1$ (median: $24 \mathrm{nmol} / 1)$ ) ${ }^{\star} \mathrm{CSF}$ was obtained from ventricular drainage. 